\title{
Periods of periodic points of 1-norm nonexpansive maps
}

\author{
Bas Lemmens \\ Eurandom, \\ P.O. Box 513, 5600 MB Eindhoven, The Netherlands \\ e-mail: lemmens@eurandom.tue.nl
}

September 2001

\section{Introduction}

In this paper several results concerning the periodic points of 1-norm nonexpansive maps will be presented. In particular, we will examine the set $R(n)$, which consists of integers $p \geq 1$ such that there exist a 1-norm nonexpansive map $f: \mathbb{R}^{n} \rightarrow \mathbb{R}^{n}$ and a periodic point of $f$ of minimal period $p$. The principal problem is to find a characterization of the set $R(n)$ in terms of arithmetical and combinatorial constraints. This problem was posed in $[12$, Section 4]. We shall present here a significant step towards such a characterization. In fact, we shall introduce for each $n \in \mathbb{N}$ a set $T(n)$ that is determined by arithmetical and combinatorial constraints only, and prove that $R(n) \subset T(n)$ for all $n \in \mathbb{N}$. Moreover, we will see that $R(n)=T(n)$ for $n=1,2,3,6,7$, and 10, but it remains an open problem whether the sets $R(n)$ and $T(n)$ are equal for all $n \in \mathbb{N}$.

Pioneering research on the periodic points of 1-norm nonexpansive maps was done by Akcoglu and Krengel. In [1] they examined the asymptotic behaviour of diffusion processes on a finite state space that can be modelled by 1-norm nonexpansive maps. As a result they showed that the asymptotic behaviour of such processes is periodic. Indeed they proved the following general theorem concerning the iterative behaviour of 1-norm nonexpansive maps.

Theorem 1.1 (cf. [1]) Let $D$ be a closed subset of $\mathbb{R}^{n}$ and let $f: D \rightarrow D$ be a 1-norm nonexpansive map. If there exists $x^{\prime} \in D$ such that $\left(\left\|f^{k}\left(x^{\prime}\right)\right\|_{1}\right)_{k}$ 
remains bounded, then for each $x \in D$ there exist an integer $p_{x}=p \geq 1$ and a periodic point $\xi_{x}=\xi \in D$ of $f$ of minimal period $p$ such that $\left(f^{k p}(x)\right)_{k}$ converges to $\xi$.

In subsequent work [7] Misiurewicz showed that for each $x \in D$ the integer $p_{x}$ in Theorem 1.1 is at most $n ! 2^{m}$, where $m=2^{n}$. Furthermore, Weller generalized in [17] Theorem 1.1 to norms on $\mathbb{R}^{n}$ for which the unit ball is a polyhedron: examples of such norms are the 1-norm and the supnorm. Thereafter various upper bounds for the integer $p_{x}$ for maps that are nonexpansive in a polyhedral norm were derived in [2], [4], [5], [6], [8], [14], and [16].

As there exists an a priori upper bound for the integer $p_{x}$ in Theorem 1.1 that only depends on the dimension of the ambient space, the set of integers $p \geq 1$ for which there exist a 1-norm nonexpansive map $f: D \rightarrow D$, with $D \subset \mathbb{R}^{n}$, and a periodic point $\xi \in D$ of $f$ of minimal period $p$, is finite. It is an interesting problem to determine this finite set for a given domain $D \subset \mathbb{R}^{n}$ explicitly.

Motivated by the models of diffusion on a finite state space one has examined in [9], [11], [12], [13], and [14] the set $P^{*}(n)$, which consists of integers $p \geq 1$ for which there exist a 1-norm nonexpansive map $f: \mathbb{K}^{n} \rightarrow$ $\mathbb{K}^{n}$, with $f(0)=0$, and a periodic point of $f$ of minimal period $p$. Here $\mathbb{K}^{n}$ denotes the positive cone in $\mathbb{R}^{n}$. Surprisingly the set $P^{*}(n)$ can be completely characterized by arithmetical and combinatorial constraints. In fact, it is shown in [11] and [12] together that $P^{*}(n)$ is precisely the set of possible periods of an admissible array on $n$ symbols, where an admissible array is defined as follows:

Definition 1.1 Let $(L,<)$ be a finite totally ordered set and let $\Sigma$ be a set with $n$ elements. For each $i \in L$ let $\vartheta_{i}: \mathbb{Z} \rightarrow \Sigma$ be a map. The sequence $\vartheta=\left(\vartheta_{i}: \mathbb{Z} \rightarrow \Sigma \mid i \in L\right)$ is called an admissible array on $n$ symbols if the maps $\vartheta_{i}$ satisfy the following properties:

(i) For each $i \in L$ there exists an integer $p_{i}$ with $1 \leq p_{i} \leq n$ such that the map $\vartheta_{i}: \mathbb{Z} \rightarrow \Sigma$ is periodic with period $p_{i}$ and moreover $\vartheta_{i}(s) \neq \vartheta_{i}(t)$ for each $1 \leq s<t \leq p_{i}$.

(ii) If $m_{1}<m_{2}<\ldots<m_{r+1}$ is an increasing sequence of distinct points in $L$ and

$$
\vartheta_{m_{i}}\left(s_{i}\right)=\vartheta_{m_{i+1}}\left(t_{i}\right) \quad \text { for } 1 \leq i \leq r,
$$


then

$$
\sum_{i=1}^{r}\left(t_{i}-s_{i}\right) \not \equiv 0 \bmod \rho, \text { where } \rho=\operatorname{gcd}\left(\left\{p_{m_{i}}: 1 \leq i \leq r+1\right\}\right) .
$$

Here $\operatorname{gcd}(S)$ denotes the greatest common divisor of the elements of the set $S$. The period of an admissible array $\vartheta=\left(\vartheta_{i}: \mathbb{Z} \rightarrow \Sigma \mid i \in L\right)$ is said to be $\operatorname{lcm}\left(\left\{p_{i}: i \in L\right\}\right)$, that is the least common multiple of the integers $p_{i}$ with $i \in L$. Therefore, if one defines for each $n \in \mathbb{N}$ :

$$
Q(n)=\{p \in \mathbb{N}: p \text { is the period of an admissible array on } n \text { symbols }\}
$$

then the characterization of the set $P^{*}(n)$ can be stated as follows:

Theorem 1.2 (cf. [12], Theorem 3.1) $P^{*}(n)=Q(n)$ for all $n \in \mathbb{N}$.

In this paper we are interested in a characterization (in terms of arithmetical and combinatorial constraints) of the set $R(n)$, which consists of the possible minimal periods of periodic points of 1-norm nonexpansive maps $f: \mathbb{R}^{n} \rightarrow \mathbb{R}^{n}$. It has been proved in [15] that $R(n) \subset P^{*}(2 n)$, so that by Theorem 1.2 the following inclusion holds:

Theorem 1.3 (cf. [12], Theorem 4.1) $R(n) \subset Q(2 n)$ for all $n \in \mathbb{N}$.

It is expected however, that the sets $R(n)$ and $Q(2 n)$ are not equal for general $n \in \mathbb{N}$. In particular, if $n=3$ it can be shown that $Q(6)=\{1,2,3,4,5,6,12\}$ (see [13]) and that $\{1,2,3,4,5,6\} \subset R(3)$, but it is conjectured in [12, page 171] that 12 is not in $R(3)$.

We will introduce for each $n \in \mathbb{N}$ a set $T(n)$ that determined by arithmetical and combinatorial constraints only, and it will be proved that $R(n) \subset$ $T(n) \subset Q(2 n)$ for each $n \in \mathbb{N}$. The inclusion $R(n) \subset T(n)$ is a significant sharpening of the inclusion in Theorem 1.3. Moreover we will see that $R(n)=T(n)$ for $n=1,2,3,4,6,7$, and 10 , but it remains open whether the two set are equal for all $n \in \mathbb{N}$. By using this equality the set $R(n)$ can be determined explicitly for $n=1,2,3,4,6,7$, and 10 . As a result we obtain the equality $R(3)=\{1,2,3,4,5,6\}$, which proves the conjecture in $[12$, page $171]$. 


\section{Main theorem}

Before we state the main theorem we recall several definitions. On $\mathbb{R}^{n}$ the 1-norm is defined by

$$
\|x\|_{1}=\sum_{i=1}^{n}\left|x_{i}\right| \quad \text { for } x=\left(x_{1}, \ldots, x_{n}\right) .
$$

A map $f: D \rightarrow \mathbb{R}^{m}$, with $D \subset \mathbb{R}^{n}$, is said to be 1 -norm nonexpansive or simply 1-nonexpansive if

$$
\|f(x)-f(y)\|_{1} \leq\|x-y\|_{1} \quad \text { for all } x, y \in D .
$$

If equality holds in (2) for every $x$ and $y$ in $D$, then $f$ is called a 1-isometry.

A point $x \in D$ is called a periodic point of $f: D \rightarrow D$ if $f^{q}(x)=x$ for some integer $q>0$, and $q$ is called a period of $x$. The smallest such $q>0$ is called the minimal period of $x$.

On $\mathbb{R}^{n}$ a partial ordering $\leq$ is defined by $x \leq y$ if $x_{i} \leq y_{i}$ for $i=1, \ldots, n$. We say that $x<y$ if $x \leq y$ and $x \neq y$. The positive cone in $\mathbb{R}^{n}$ is said to be $\mathbb{K}^{n}=\left\{x \in \mathbb{R}^{n}: x \geq 0\right\}$. Further for each $x, y \in \mathbb{R}^{n}$ we let $x \vee y$ denote the least upper bound of $x$ and $y$ in $\mathbb{R}^{n}$, so $(x \vee y)_{i}=\max \left\{x_{i}, y_{i}\right\}$ for $i=1, \ldots, n$. Similarly, $x \wedge y$ denotes the greatest lower bound of $x$ and $y$ in $\mathbb{R}^{n}$, so $(x \wedge y)_{i}=\min \left\{x_{i}, y_{i}\right\}$ for $i=1, \ldots, n$. A map $f: D \rightarrow \mathbb{R}^{m}$, with $D \subset \mathbb{R}^{n}$, is called order-preserving if $f(x) \leq f(y)$ for all $x, y \in D$ with $x \leq y$.

Several sets of integers occur frequently in the exposition and for convenience we list them in the following definition.

Definition 2.1 For each $n \in \mathbb{N}$ :

(i) $R(n)$ is said to be the set of integers $p \geq 1$ such that there exist a 1-nonexpansive map $f: \mathbb{R}^{n} \rightarrow \mathbb{R}^{n}$ and a periodic point of $f$ of minimal period $p$.

(ii) $P^{*}(n)$ is said to be the set of integers $p \geq 1$ such that there exist a 1-nonexpansive map $f: \mathbb{K}^{n} \rightarrow \mathbb{K}^{n}$, with $f(0)=0$, and a periodic point of minimal period $p$.

To state the main theorem the idea of a strongly admissible array on $2 n$ symbols is needed. However, before a definition of a strongly admissible array can be given a piece of notation is required. If $a \in\{1,2, \ldots, 2 n\}$, then we write $a^{+}=a+n$ if $1 \leq a \leq n$, and $a^{+}=a-n$ if $n+1 \leq a \leq 2 n$. 
Definition 2.2 Let $(L,<)$ be a finite totally ordered set and let $\Sigma=$ $\{1,2, \ldots, 2 n\}$. Assume that $\vartheta=\left(\vartheta_{i}: \mathbb{Z} \rightarrow \Sigma \mid i \in L\right)$ is an admissible array on $2 n$ symbols, and let $p_{i}$ be the period of $\vartheta_{i}$ for $i \in L$. We call $\vartheta$ a strongly admissible array on $2 n$ symbols if the maps $\vartheta_{i}$ satisfy:

(i) If $m_{1}, m_{2}$ are distinct elements of $L$ and $\vartheta_{m_{1}}(s)=\vartheta_{m_{2}}(t)^{+}$, then

$$
t-s \not \equiv 0 \bmod \pi \text {, where } \pi=\operatorname{gcd}\left(\left\{p_{m_{1}}, p_{m_{2}}\right\}\right) \text {. }
$$

(ii) Let $m_{1}<m_{2}<\ldots<m_{r+1}$ be an increasing sequence of distinct points in $L$. If

$$
\vartheta_{m_{i}}\left(s_{i}\right)=\vartheta_{m_{i+1}}\left(t_{i}\right) \quad \text { for } 1 \leq i \leq r
$$

and $\vartheta_{m_{1}}(u)=\vartheta_{m_{r+1}}(v)^{+}$, then

$$
\sum_{i=1}^{r}\left(t_{i}-s_{i}\right) \not \equiv(v-u) \bmod \rho \text {, where } \rho=\operatorname{gcd}\left(\left\{p_{m_{i}}: 1 \leq i \leq r+1\right\}\right) \text {. }
$$

Again the period of a strongly admissible array $\vartheta=\left(\vartheta_{i}: \mathbb{Z} \rightarrow \Sigma \mid i \in L\right)$ is said to be $\operatorname{lcm}\left(\left\{p_{i}: i \in L\right\}\right)$. Now for each $n \in \mathbb{N}$ we define

$T(n)=\{p \geq 1: p$ is the period of a strongly admissible array on $2 n$ symbols $\}$,

and we state the main theorem as follows:

Theorem 2.1 $R(n) \subset T(n)$ for all $n \in \mathbb{N}$.

It is clear from the definition of $T(n)$ that $T(n) \subset Q(2 n)$, and moreover it will be shown that $T(n)$ is strictly smaller than $Q(2 n)$ for all $n \geq 3$, so that Theorem 2.1 is a sharpening of the inclusion $R(n) \subset Q(2 n)$ in Theorem 1.3.

The proof of Theorem 2.1 is based on the following ideas. For every $p \in R(n)$ there exist a 1-nonexpansive map $f: \mathbb{K}^{2 n} \rightarrow \mathbb{K}^{2 n}$, with $f(0)=0$, and a periodic point $\xi \in \mathbb{K}^{2 n}$ of $f$ of minimal period $p$. This follows (as we will see in Section 3) from the proof of the inclusion $R(n) \subset P^{*}(2 n)$. It turns out that $\xi$ is in a set $S \subset \mathbb{K}^{2 n}$ that is left invariant by $f$, and for each $(x, y) \in S$, with $x, y \in \mathbb{K}^{n}$, one has that $x \wedge y=0$. This geometric property of the points $f^{j}(\xi)$, where $0 \leq j<p$, is used to prove that the admissible array that can be associated with the periodic point $\xi$ of $f$ is in fact a strongly admissible array on $2 n$ symbols. 
The remainder of the paper is organized as follows: In Section 3 we recall some results from the literature that are used in the proof of Theorem 2.1. In particular, the ideas behind the inclusions $R(n) \subset P^{*}(2 n)$ and $P^{*}(n) \subset Q(n)$ are discussed. Subsequently the proof of Theorem 2.1 is given in Section 4 . In Section 5 several properties of strongly admissible arrays are derived. These properties are then used in Section 6 to make several remarks about the optimality of the upper estimate $T(n)$ for $R(n)$. The final section is used for a discussion of several open problems and some concluding remarks.

\section{Background material}

In this section results from [11] and [14] by Nussbaum and Scheutzow are recalled. These results are needed for the proof of Theorem 2.1. The bigger part of the section is devoted to the ideas behind the proof of the inclusion $P^{*}(n) \subset Q(n)$. But we start by discussing a relation between the sets $R(n)$ and $P^{*}(n)$.

\subsection{Relation with nonnegative 1-nonexpansive maps}

It is shown in [15], but also in [10, Example 3], that $R(n) \subset P^{*}(2 n)$ for all $n \geq 1$. The proof of this inclusion will be important in the sequel and therefore we discuss it in detail here.

Theorem 3.1 (cf. [15]) $R(n) \subset P^{*}(2 n)$ for all $n \in \mathbb{N}$.

Proof. Let $p \in R(n)$. By definition there exist a 1-nonexpansive map $f$ : $\mathbb{R}^{n} \rightarrow \mathbb{R}^{n}$ and a periodic point $\xi \in \mathbb{R}^{n}$ of $f$ of minimal period $p$.

First it is shown that $f$ has a fixed point. Put $S=\left\{f^{j}(\xi): 0 \leq j<p\right\}$ and let $d=\max \left\{\|x-y\|_{1}: x, y \in S\right\}$. For each $x \in \mathbb{R}^{n}$ let $B(x, d)$ denote the closed ball with radius $d$ around $x$ and put $D=\cap_{x \in S} B(x, d)$. Clearly $S \subset D$, so that $D$ is a nonempty, convex, and compact subset of $\mathbb{R}^{n}$. As $f$ is 1-nonexpansive $f[B(x, d)] \subset B(f(x), d)$ for each $x \in S$. Combining this with $f[S]=S$ yields $f[D] \subset D$. Now by application of the Brouwer fixed point theorem $f$ has a fixed point, say $x^{*} \in \mathbb{R}^{n}$.

Proceed by defining a map $f^{*}: \mathbb{R}^{n} \rightarrow \mathbb{R}^{n}$ by $f^{*}(x)=f\left(x+x^{*}\right)-x^{*}$ for $x \in \mathbb{R}^{n}$. The map $f^{*}$ is 1 -nonexpansive, $f^{*}(0)=0$, and $\xi-x^{*}$ is a periodic point of $f^{*}$ of minimal period $p$.

Let $\mathbb{E}^{2 n} \subset \mathbb{K}^{2 n}$ be defined by $\mathbb{E}^{2 n}=\left\{(x, y) \in \mathbb{K}^{n} \times \mathbb{K}^{n}: x \wedge y=0\right\}$ and consider the 1-isometry $J: \mathbb{R}^{n} \rightarrow \mathbb{E}^{2 n}$ given by $J(x)=(x \vee 0,(-x) \vee 0)$ for $x \in \mathbb{R}^{n}$. Since $J$ is onto it has an inverse $J^{-1}: \mathbb{E}^{2 n} \rightarrow \mathbb{R}^{n}$ and $J^{-1}$ is also 
a 1-isometry. Now let $g: \mathbb{E}^{2 n} \rightarrow \mathbb{E}^{2 n}$ be given by $g(z)=\left(J \circ f^{*} \circ J^{-1}\right)(z)$ for $z \in \mathbb{E}^{2 n}$. The map $g$ is by construction 1-nonexpansive and $g(0)=0$. Moreover, $J\left(\xi-x^{*}\right) \in \mathbb{E}^{2 n}$ is a periodic point of $g$ of minimal period $p$.

Now we arrive at the final step in the proof. Define $R: \mathbb{K}^{2 n} \rightarrow \mathbb{E}^{2 n}$ by

$$
R((x, y))=(x-(x \wedge y), y-(x \wedge y)) \quad \text { for } x, y \in \mathbb{K}^{n} .
$$

It is not hard to verify that $R$ is 1-nonexpansive, and $R(z)=z$ for all $z \in \mathbb{E}^{2 n}$. Using the map $R$ we finally define $h: \mathbb{K}^{2 n} \rightarrow \mathbb{K}^{2 n}$ by

$$
h(z)=\left(J \circ f^{*} \circ J^{-1} \circ R\right)(z) \quad \text { for } z \in \mathbb{K}^{2 n} .
$$

By construction, $h$ is 1-nonexpansive and $h(0)=0$. As $R(z)=z$ for all $z \in \mathbb{E}^{2 n}$, we find that $J\left(\xi-x^{*}\right) \in \mathbb{E}^{2 n}$ is a periodic point of $h$ of minimal period $p$. Therefore $p \in P^{*}(2 n)$ and this proves the theorem.

From the proof of Theorem 3.1 we can extract the following assertion.

Corollary 3.1 For each $p \in R(n)$ there exist a 1-nonexpansive map $f$ : $\mathbb{K}^{2 n} \rightarrow \mathbb{K}^{2 n}$, with $f(0)=0$, and a periodic point $\xi \in \mathbb{K}^{2 n}$ of $f$ of minimal period $p$. Moreover, for each $0 \leq j<p$ the point $f^{j}(\xi)$ is in $\mathbb{E}^{2 n}=\{(x, y) \in$ $\left.\mathbb{K}^{n} \times \mathbb{K}^{n}: x \wedge y=0\right\}$.

\subsection{Periods of nonnegative 1-nonexpansive maps}

The set $P^{*}(n)$ has been studied intensively in [1], [9], [11], [12], [13], and [14]. As a major result it is shown in [11] and [12] together that $P^{*}(n)=Q(n)$ for all $n \geq 1$. Here $Q(n)$ is the set of periods of admissible arrays on $n$ symbols, as defined in (1). The main objective of this subsection is to explain the ideas behind the proof of the inclusion $P^{*}(n) \subset Q(n)$. We begin by collecting several definitions.

A set $V \subset \mathbb{R}^{n}$ is called a lower semilattice if $x \in V$ and $y \in V$ implies $x \wedge y \in V$. For $S \subset \mathbb{R}^{n}$ there exists a minimal (in the sense of inclusion) lower semilattice $V_{S} \supset S$. The set $V_{S}$ will be called the lower semilattice generated by $S$. Remark that if $S$ is finite, so is $V_{S}$. If $V \subset \mathbb{R}^{n}$ is a lower semilattice and $g: V \rightarrow V$ is such that

$$
g(x \wedge y)=g(x) \wedge g(y) \quad \text { for all } x, y \in V,
$$

then $g$ is called a lower semilattice homomorphism.

If $V \subset \mathbb{R}^{n}$ is a lower semilattice and $g: V \rightarrow V$ is a lower semilattice homomorphism, then $x, y \in V$ and $x \leq y$ implies $g(x)=g(x \wedge y)=g(x) \wedge$ 
$g(y)$, so that $g(x) \leq g(y)$. Thus, every lower semilattice homomorphism is order preseving. Furthermore, if $\xi \in V$ is a periodic point of a lower semilattice homomorphism $g: V \rightarrow V$ of minimal period $p$, and $U$ is the lower semilattice generated by $\left\{g^{j}(\xi): 0 \leq j<p\right\}$, then $g^{p}(x)=x$ for all $x \in U$, and $g[U]=U$. Therefore, the restriction of $g$ to $U$ is a bijective lower semilattice homomorphism, with inverse $g^{-1}=g^{p-1}$. Using these definitions and remarks we can now state the following theorem (see [14, Lemma 3.2]).

Theorem 3.2 Let $f: \mathbb{K}^{n} \rightarrow \mathbb{K}^{n}$ be a 1-nonexpansive map, $f(0)=0$, and $\xi \in \mathbb{K}^{n}$ be a periodic point of $f$ of minimal period $p$. Let $V \subset \mathbb{K}^{n}$ denote the lower semilattice generated by $\left\{f^{j}(\xi): 0 \leq j<p\right\}$ and let $g=f_{\mid V}$. Then the map $g$ is a lower semilattice homomorphism that maps $V$ onto itself.

Theorem 3.2 plays a key role in the proof of the inclusion $P^{*}(n) \subset Q(n)$ and it motivates a further analysis of homomorphisms on a finite lower semilattice.

Let $V$ be a finite lower semilattice in $\mathbb{R}^{n}$ and let $A \subset V$. If there exists $\beta \in V$ such that $\alpha \leq \beta$ for each $\alpha \in A$, we say that $A$ is bounded above in $V$, and $\beta$ is called an upper bound of $A$ in $V$. Lower bounds can be defined in the same manner (with $\geq$ in place of $\leq$ ). If $A$ is bounded above in $V$, then there exists a unique $\alpha \in V$ upper bound of $A$ in $V$ such that $\gamma<\alpha$ implies $\gamma$ is not an upper bound of $A$ in $V$. The element $\alpha \in V$ will be called the supremum of $A$ in $V$ and we write $\alpha=\sup _{V}(A)$. Analogously we define the infimum of $A$ in $V$, denoted $\inf _{V}(A)$, to be the unique lower bound $\alpha \in V$ of $A$ such that no $\beta>\alpha$ is a lower bound of $A$ in $V$. Since $V \subset \mathbb{R}^{n}$ we see that $\alpha=\bigwedge\{a: a \in A\}$ is the infimum of $A$ in $V$. Furthermore, if $A$ is bounded above in $V$ and we let

$$
B_{A}=\{b \in V: b \text { is an upper bound of } A \text { in } V\},
$$

then $\sup _{V}(A)=\inf _{V}\left(B_{A}\right)$.

For each $x \in V$ the height of $x$ in $V$, denoted $h_{V}(x)$, is defined by

$$
\begin{aligned}
& h_{V}(x)=\sup \left\{k \geq 0: \text { there exist } y^{0}, \ldots, y^{k} \in V\right. \text { such that } \\
& \left.\qquad y^{k}=x \text { and } y^{j}<y^{j+1} \text { for } 0 \leq j<k\right\}<\infty .
\end{aligned}
$$

If no $y \in V$ exists with $y<x$, then we put $h_{V}(x)=0$. Note that there is only one element in $V$ with $h_{V}(x)=0$, namely $\inf _{V}(V)$. For every $x \in V$ we define $S_{x}=\{y \in V: y<x\}$. An element $x \in V$ is called irreducible in $V$ if either $S_{x}$ is empty or

$$
x>\sup _{V}\left(S_{x}\right) .
$$


If $x \in V$ is irreducible in $V, S_{x}$ is nonempty, and $z$ denotes the $\sup _{V}\left(S_{x}\right)$, then we define $I_{V}(x)$ by

$$
I_{V}(x)=\left\{i: x_{i}>z_{i}\right\}
$$

In case $S_{x}$ is empty, that is to say $x$ is the infimum of $V$, then we put $I_{V}(x)=\{1,2, \ldots, n\}$. Remark that if $x \in V$ is irreducible in $V, S_{x}$ is nonempty, and $\bar{x}$ denotes the infimum of $V$, then

$$
x_{i}>\bar{x}_{i} \text { for all } i \in I_{V}(x) .
$$

In relation with these definitions there is a relevant result of Scheutzow [14, Lemma 3.2]. A proof of this version of the lemma can be found in [11, Lemma 1.1].

Lemma 3.1 Let $j \in \mathbb{Z}$, let $V$ be a finite lower semilattice in $\mathbb{R}^{n}$, and let $f: V \rightarrow V$ be a one-to-one lower semilattice homomorphism of $V$ onto itself. If $y \in V$ and $f^{j}(y) \neq y$, then $y$ and $f^{j}(y)$ are incomparable, and $h_{V}(y)=h_{V}\left(f^{j}(y)\right)$, where $h_{V}(\cdot)$ is the height function given in (6). If $y$ is irreducible in $V$, then $f^{j}(y)$ is irreducible in $V$. If $\eta \in V$ and $\zeta \in V$ are not comparable, and $\eta$ and $\zeta$ are irreducible in $V$, then

$$
I_{V}(\eta) \cap I_{V}(\zeta)=\emptyset .
$$

If $y \in V$ is irreducible in $V$ and $y$ is a periodic point of $f$ of minimal period $p$, then $1 \leq p \leq n$.

We now give a technical definition. This definition forms the base from which an admisssible array can be constructed.

Definition 3.1 Let $W$ be a lower semilattice in $\mathbb{R}^{n}$, let $g: W \rightarrow W$ be a lower semilattice homomorphism, and let $\xi \in W$ be a periodic point of $g$ of minimal period $p$. Let $V$ denote the lower semilattice generated by $\left\{g^{j}(\xi): j \geq 0\right\}$ and $f=g_{\mid V}$. A finite sequence $\left(y^{i}\right)_{i=1}^{m} \subset V$ is called a complete sequence for $\xi$, if it satisfies:

(i) For $i=1, \ldots, m$ we have $y^{i} \leq \xi$.

(ii) For $i=1, \ldots, m$ the element $y^{i}$ is irreducible in $V$.

(iii) If $p_{i}$ denotes the minimal period of $y^{i}$ under $f$, then $p=\operatorname{lcm}\left(\left\{p_{i}: 1 \leq\right.\right.$ $i \leq m\})$.

(iv) For $1 \leq i<m$ we have $h_{V}\left(y^{i}\right) \leq h_{V}\left(y^{i+1}\right)$, where $h_{V}(\cdot)$ is the height function given by equation (6). 
(v) For $1 \leq i<j \leq m$, the sets $\left\{f^{k}\left(y^{i}\right): k \geq 0\right\}$ and $\left\{f^{k}\left(y^{j}\right): k \geq 0\right\}$ are disjoint.

(vi) For $1 \leq i<j \leq m$, the elements $y^{i}$ and $y^{j}$ are not comparable.

The following result says that every periodic point of a lower semilattice homomorphism has a complete sequence (see [11, Proposition 1.1]).

Proposition 3.1 If $W$ is a lower semilattice in $\mathbb{R}^{n}, g: W \rightarrow W$ is a lower semilattice homomorphism, and $\xi \in W$ is a periodic point of $g$, then there exists a complete sequence for $\xi$.

From a complete sequence one can now construct an admissible array. Let $W$ be a lower semilattice in $\mathbb{R}^{n}$ and let $g: W \rightarrow W$ be a lower semilattice homomorphism. Suppose that $\xi \in W$ is a periodic point of $g$ of minimal period $p$. Let $V$ denote the lower semilattice generated by $\left\{g^{j}(\xi): j \geq 0\right\}$ and put $f=g_{\mid V}$. Now by Proposition 3.1 there exists a complete sequence $\left(y^{i}\right)_{i=1}^{m} \subset V$ for $\xi$. It follows from property (ii) in Definition 3.1 and Lemma 3.1 that $f^{j}\left(y^{i}\right)$ is irreducible in $V$ for $1 \leq i \leq m$ and $j \in \mathbb{Z}$. Therefore the set $I_{V}\left(f^{j}\left(y^{i}\right)\right.$ ) (as defined in (8)) is nonempty for $1 \leq i \leq m$ and $j \in \mathbb{Z}$. Let $p_{i}$ denote the minimal period of $y^{i}$ under $f$. Select for $i=1, \ldots, m$ and $j=0, \ldots, p_{i}-1$ an integer $a_{i j} \in I_{V}\left(f^{j}\left(y^{i}\right)\right)$. Furthermore, define for $i=1, \ldots, m$ and general $j \in \mathbb{Z}$ the integer $a_{i j}$ by

$$
a_{i j}=a_{i k}, \text { where } 0 \leq k<p_{i} \text { and } j \equiv k \bmod p_{i} .
$$

The semi-infinite matrix $\left(a_{i j}\right)$, where $i=1, \ldots, m$ and $j \in \mathbb{Z}$, is called an array of $\xi$. Now one can prove the following connection with the admissible arrays on $n$ symbols (see [11, Propostion 1.2]).

Proposition 3.2 Let $W$ be a lower semilattice in $\mathbb{R}^{n}, g: W \rightarrow W$ be a lower semilattice homomorphism, and $\xi \in W$ be a periodic point of $g$ of minimal period $p$. Let $\left(a_{i j}\right)$, where $i=1, \ldots, m$ and $j \in \mathbb{Z}$, be an array of $\xi$. Further, let $L=\{1, \ldots, m\}$ be equipped with the usual ordering and $\Sigma=\{1,2, \ldots, n\}$. If $\vartheta=\left(\vartheta_{i}: \mathbb{Z} \rightarrow \Sigma \mid i \in L\right)$ is defined by

$$
\vartheta_{i}(j)=a_{i j} \quad \text { for } i \in L \text { and } j \in \mathbb{Z},
$$

then $\vartheta$ is an admissible array on $n$ symbols of period $p$.

A combination of Theorem 3.2, Proposition 3.1, and Proposition 3.2 yields the inclusion $P^{*}(n) \subset Q(n)$ for all $n \in \mathbb{N}$. The other inclusion $Q(n) \subset$ $P^{*}(n)$ is proved in [12]. 
Now let us go back to the set $R(n)$. From Corollary 3.1 it follows that for every $p \in R(n)$ there exist a 1-nonexpansive map $f: \mathbb{K}^{2 n} \rightarrow \mathbb{K}^{2 n}$, with $f(0)=0$, and a periodic point $\xi \in \mathbb{K}^{2 n}$ of $f$ of minimal period $p$. Furthermore, $f^{j}(\xi)$ is in $\mathbb{E}^{2 n}$ for each $0 \leq j<p$, so that the lower semilattice generated by $\left\{f^{j}(\xi): 0 \leq j<p\right\}$ is contained in $\mathbb{E}^{2 n}$. This follows from the fact that $\mathbb{E}^{2 n}$ is itself a lower semilattice. Therefore it makes sense (in the light of the results in this section) to study the arrays of periodic points of lower semilattice homomorphisms $g: W \rightarrow W$ with $W \subset \mathbb{E}^{2 n}$.

\section{Proof of the main theorem}

In this section we prove the main result: Theorem 2.1. The principal idea is to show that the arrays of periodic points of lower semillatice homomorphisms $g: W \rightarrow W$, with $W \subset \mathbb{E}^{2 n}$, give rise to strongly admissible arrays on $2 n$ symbols.

Proposition 4.1 Let $W$ be a lower semilattice in $\mathbb{E}^{2 n}, g: W \rightarrow W$ be a lower semilattice homomorphism, and $\xi \in W$ be a periodic point of $g$ of minimal period $p$. Let $\left(a_{i j}\right)$, where $1 \leq i \leq m$ and $j \in \mathbb{Z}$, be an array of $\xi$. Further, let $L=\{1, \ldots, m\}$ be equipped with the usual ordering and $\Sigma=\{1, \ldots, 2 n\}$. If $\vartheta=\left(\vartheta_{i}: \mathbb{Z} \rightarrow \Sigma \mid i \in L\right)$ is defined by

$$
\vartheta_{i}(j)=a_{i j} \quad \text { for } i \in L \text { and } j \in \mathbb{Z},
$$

then $\vartheta$ is a strongly admissible array on $2 n$ symbols of period $p$.

Proof. Let $W$ be a lower semilattice in $\mathbb{E}^{2 n}, g: W \rightarrow W$ be a lower semilattice homomorphism, and $\xi \in W$ be a periodic point of $g$ of minimal period $p$. Furthermore, let $V$ denote the lower semilattice generated by $\left\{g^{j}(\xi): j \geq 0\right\}$ and $f=g_{\mid V}$. Suppose that $\left(a_{i j}\right)$, where $1 \leq i \leq m$ and $j \in \mathbb{Z}$, is an array of $\xi$. Let $\left(y_{i}\right)_{i=1}^{m} \subset V$ be a complete sequence for $\xi$ that induces the array $\left(a_{i j}\right)$. For $i=1, \ldots, m$ let $p_{i}$ denote the minimal period of $y^{i}$ under $f$, so $p=\operatorname{lcm}\left(\left\{p_{i}: 1 \leq i \leq m\right\}\right)$ by property (iii) in Definition 3.1. Further, put $\Sigma=\{1,2, \ldots, 2 n\}$ and let $L=\{1, \ldots, m\}$ be equipped with the usual ordering. Assume that $\vartheta=\left(\vartheta_{i}: \mathbb{Z} \rightarrow \Sigma \mid i \in L\right)$ is defined by

$$
\vartheta_{i}(j)=a_{i j} \quad \text { for } i \in L \text { and } j \in \mathbb{Z} \text {. }
$$

By construction we have that $\vartheta_{i}(j)=a_{i j} \in I_{V}\left(f^{j}\left(y^{i}\right)\right)$ for $i=1, \ldots, m$ and $j \in \mathbb{Z}$. Moreover it follows from Proposition 3.2 that $\vartheta$ is an admissible array on $2 n$ symbols. As $\vartheta_{i}$ has period $p_{i}$ for $1 \leq i \leq m$ and $p=\operatorname{lcm}\left(\left\{p_{i}\right.\right.$ : 
$1 \leq i \leq m\}$ ) the admissible array has period $p$. Therefore it suffices to show that the maps $\vartheta_{i}$ with $i \in L$, satisfy the two properties in Definition 2.2. For both properties we will give an argument by contradiction. Let us start with the first one.

Suppose that there exist $s, t \in \mathbb{Z}$ and distinct $m_{1}, m_{2} \in L$ such that $\vartheta_{m_{1}}(s)=\vartheta_{m_{2}}(t)^{+}$and $(t-s) \equiv 0 \bmod \pi$, where $\pi=\operatorname{gcd}\left(\left\{p_{m_{1}}, p_{m_{2}}\right\}\right)$. As a first step it is shown that there exists $w \in \mathbb{Z}$ such that $\vartheta_{m_{1}}(w)=\vartheta_{m_{2}}(w)^{+}$.

From $(t-s) \equiv 0 \bmod \pi$ it follows that there exists an integer $q$ such that $(t-s)-q \pi=0$. Furthermore, since $\pi=\operatorname{gcd}\left(\left\{p_{m_{1}}, p_{m_{2}}\right\}\right)$ there exist $k_{1}, k_{2} \in \mathbb{Z}$ such that $k_{1} p_{m_{1}}+k_{2} p_{m_{2}}=\pi$. Therefore we obtain that

$$
(t-s)-q\left(k_{1} p_{m_{1}}+k_{2} p_{m_{2}}\right)=0 .
$$

Now we put $w=s+q k_{1} p_{m_{1}}$. As $\vartheta_{m_{1}}$ and $\vartheta_{m_{2}}$ have period $p_{m_{1}}$ and $p_{m_{2}}$ respectively, we have that $\vartheta_{m_{1}}(k)=\vartheta_{m_{1}}\left(k+p_{m_{1}}\right)$ and $\vartheta_{m_{2}}(k)=\vartheta_{m_{2}}\left(k+p_{m_{2}}\right)$ for all $k \in \mathbb{Z}$. Therefore (11) gives $\vartheta_{m_{1}}(w)=\vartheta_{m_{2}}(w)^{+}$.

To continue the proof remark that

$$
\vartheta_{m_{1}}(w) \in I_{V}\left(f^{w}\left(y^{m_{1}}\right)\right) \quad \text { and } \quad \vartheta_{m_{2}}(w) \in I_{V}\left(f^{w}\left(y^{m_{2}}\right)\right) .
$$

It follows from property (i) in Definition 3.1 that $y^{m_{1}} \leq \xi$ and $y^{m_{2}} \leq \xi$. As $f$ is a lower semilattice homomorphism it is order preserving on $V$, so that

$$
f^{w}\left(y^{m_{1}}\right) \leq f^{w}(\xi) \quad \text { and } \quad f^{w}\left(y^{m_{2}}\right) \leq f^{w}(\xi) .
$$

Let $\bar{x}=\inf _{V}(V)$. Since $V$ is a subset of $\mathbb{E}^{2 n}$ it follows that $\bar{x} \geq 0$. In combination with (9), (12) and (13) this gives:

$$
0 \leq \bar{x}_{\vartheta_{m_{1}}(w)}<f^{w}\left(y^{m_{1}}\right)_{\vartheta_{m_{1}}(w)} \leq f^{w}(\xi)_{\vartheta_{m_{1}}(w)}
$$

and

$$
0 \leq \bar{x}_{\vartheta_{m_{2}}(w)}<f^{w}\left(y^{m_{2}}\right)_{\vartheta_{m_{2}}(w)} \leq f^{w}(\xi)_{\vartheta_{m_{2}}(w)} .
$$

The equality $\vartheta_{m_{1}}(w)=\vartheta_{m_{2}}(w)^{+}$together with (14) and (15) imply

$$
f^{w}(\xi)_{\vartheta_{m_{2}}(w)^{+}} \cdot f^{w}(\xi)_{\vartheta_{m_{2}}(w)}>0 .
$$

This, however, contradicts the fact that $f^{j}(\xi) \in \mathbb{E}^{2 n}$ for every $j \in \mathbb{Z}$.

To show the second property, let $m_{1}<m_{2}<\ldots<m_{r+1}$ be an increasing sequence of $(r+1)$ distinct elements in $L$ such that

$$
\vartheta_{m_{i}}\left(s_{i}\right)=\vartheta_{m_{i+1}}\left(t_{i}\right) \quad \text { for } 1 \leq i \leq r,
$$


and assume that there exist $u, v \in \mathbb{Z}$ such that $\vartheta_{m_{1}}(u)=\vartheta_{m_{r+1}}(v)^{+}$. Further, suppose by way of contradiction that

$$
\sum_{i=1}^{r}\left(t_{i}-s_{i}\right) \equiv(v-u) \quad \bmod \rho, \quad \text { where } \rho=\operatorname{gcd}\left(\left\{p_{m_{i}}: i \in L\right\}\right) .
$$

From elementary properties of the greatest common divisor it follows that

$$
\rho=\sum_{i=1}^{r+1} A_{i} p_{m_{i}} \quad \text { for certain integers } A_{1}, A_{2}, \ldots, A_{r+1} .
$$

Therefore there exist integers $B_{1}, B_{2}, \ldots, B_{r+1}$ such that

$$
\sum_{i=1}^{r}\left(t_{i}-s_{i}\right)-\sum_{i=1}^{r+1} B_{i} p_{m_{i}}=v-u \text {. }
$$

Let $\eta^{i}=f^{s_{i}}\left(y^{m_{i}}\right)$ and $\zeta^{i}=f^{t_{i}}\left(y^{m_{i+1}}\right)$ for all $i=1, \ldots, r$. Property (ii) and (v) in Definition 3.1 imply that $\eta^{i}, \zeta^{i}$ are irreducible in $V$, and $\eta^{i} \neq \zeta^{i}$ for $1 \leq i \leq r$. As $\vartheta_{m_{i}}\left(s_{i}\right)=\vartheta_{m_{i+1}}\left(t_{i}\right)$, it follows that $I_{V}\left(\eta^{i}\right)$ and $I_{V}\left(\zeta^{i}\right)$ have a nonempty intersection. Therefore, we can conclude from Lemma 3.1 that $\eta^{i}$ and $\zeta^{i}$ are comparable for $1 \leq i \leq r$. Since $m_{i}<m_{i+1}$ we obtain by property (iv) of Definition 3.1 that $h_{V}\left(\eta^{i}\right) \leq h_{V}\left(\zeta^{i}\right)$, and hence $\eta^{i}<\zeta^{i}$ for $1 \leq i \leq r$.

Using the fact that $f^{j}$ is order preserving on $V$ for each $j \in \mathbb{Z}$, and $f^{B_{i} p_{m_{i}}}\left(y^{m_{i}}\right)=y^{m_{i}}$ now gives:

$$
y^{m_{i}}<f^{\left(t_{i}-s_{i}\right)-B_{i} p_{m_{i}}}\left(y^{m_{i+1}}\right) .
$$

By repeatedly applying (17) and recalling that $f^{j}$ is order preserving on $V$ for each $j \in \mathbb{Z}$ we obtain:

$$
y^{m_{1}}<f^{\nu}\left(y^{m_{r+1}}\right), \quad \text { where } \nu=\sum_{i=1}^{r}\left(t_{i}-s_{i}\right)-\sum_{i=1}^{r} B_{i} p_{m_{i}} .
$$

Let $\mu=-B_{r+1} p_{m_{r+1}}$ and remark that $f^{\mu}\left(y^{m_{r+1}}\right)=y^{m_{r+1}}$. It then follows from a combination of (16) and (18) that

$$
y^{m_{1}}<f^{\nu+\mu}\left(y^{m_{r+1}}\right)=f^{v-u}\left(y^{m_{r+1}}\right),
$$

and therefore

$$
f^{u}\left(y^{m_{1}}\right)<f^{v}\left(y^{m_{r+1}}\right)
$$


On the other hand, as $\vartheta_{m_{1}}(u) \in I_{V}\left(f^{u}\left(y^{m_{1}}\right)\right)$ and $\vartheta_{m_{r+1}}(v) \in I_{V}\left(f^{v}\left(y^{m_{r+1}}\right)\right)$ we can apply (9) and observe that

$$
0<f^{u}\left(y^{m_{1}}\right)_{\vartheta_{m_{1}}(u)} \quad \text { and } \quad 0<f^{v}\left(y^{m_{r+1}}\right)_{\vartheta_{m_{r+1}}(v)} .
$$

Since $f^{v}\left(y^{m_{r+1}}\right)$ is an element of $\mathbb{E}^{2 n}$, the last inequality in (20) implies that

$$
f^{v}\left(y^{m_{r+1}}\right)_{\vartheta_{m_{r+1}}(v)^{+}}=0 .
$$

If we now use the assumption that $\vartheta_{m_{1}}(u)=\vartheta_{m_{r+1}}(v)^{+}$, then it follows from (20) and (21) that

$$
0=f^{v}\left(y^{m_{r+1}}\right)_{\vartheta_{m_{1}}(u)}<f^{u}\left(y^{m_{1}}\right)_{\vartheta_{m_{1}}(u)} .
$$

This contradicts inequality (19), and therefore the second property holds.

Using this proposition we can now prove Theorem 2.1.

Proof. [Proof of Theorem 2.1.] To prove the inclusion $R(n) \subset T(n)$ for all $n \in \mathbb{N}$, we take $p \in R(n)$. By Corollary 3.1 there exist a 1-nonexpansive map $f: \mathbb{K}^{2 n} \rightarrow \mathbb{K}^{2 n}$, with $f(0)=0$, and a periodic point $\xi \in \mathbb{K}^{2 n}$ of $f$ of minimal period $p$. Moreover $f^{j}(\xi) \in \mathbb{E}^{2 n}$ for each $0 \leq j<p$. Let $W$ be the lower semilattice generated by $\left\{f^{j}(\xi): 0 \leq j<p\right\}$ and let $g$ be the restriction of $f$ to $W$. As $f^{j}(\xi)$ is in $\mathbb{E}^{2 n}$ for each $0 \leq j<p$, and $\mathbb{E}^{2 n}$ is a lower semilattice: $W \subset \mathbb{E}^{2 n}$. Further, by Theorem 3.2 the map $g$ is a lower semillatice homomorphism that maps $W$ onto itself, and $\xi$ is a periodic point of $g$ of minimal period $p$. Therefore we can apply Proposition 4.1 to conclude that $p$ is the period of a strongly admissible array on $2 n$ symbols, and this proves the inclusion $R(n) \subset T(n)$.

Now that we know that the set $T(n)$ is an upper estimate of the set $R(n)$ it is natural to ask how well $T(n)$ approximates $R(n)$. To get an idea one can compute the set $T(n)$ and then one can try to find for each $p \in T(n)$ a 1nonexpansive map $f: \mathbb{R}^{n} \rightarrow \mathbb{R}^{n}$, that has a periodic point of minimal period $p$. As the definition of a strongly admissible array is rather complicated, it is difficult to compute the set $T(n)$. In the next section we discuss several properties of strongly admissible arrays that will help us to determine $T(n)$ for small $n$.

\section{Properties of strongly admissible arrays}

We begin by introducing some notation. Let $\Sigma=\{1, \ldots, 2 n\}$ and $\vartheta: \mathbb{Z} \rightarrow \Sigma$ be a map. Then we define $R(\vartheta)=\{\vartheta(s): s \in \mathbb{Z}\}$, and for every $U \subset \Sigma$ we write $U^{+}=\left\{u^{+}: u \in U\right\}$ and $\bar{U}=U \cup U^{+}$. 
To facilitate the analysis of the set $T(n)$ we shall write it in a different way, and therefore the following concept is introduced.

Definition 5.1 Let $S=\left\{q_{i}: i=1, \ldots, m\right\}$ be a set of distinct integers between 1 and $2 n$. The set $S$ is called strongly array admissible for $2 n$, if there exist a totally ordered set $(L,<)$ of $m$ elements, a strongly admissible array $\vartheta=\left(\vartheta_{i}: \mathbb{Z} \rightarrow \Sigma \mid i \in L\right)$ on $2 n$ symbols, where $\vartheta_{i}$ has period say $p_{i}$ for $i \in L$, and there exists a one-to-one map $\sigma$ of $\{1,2, \ldots, m\}$ onto $L$ such that $q_{i}=p_{\sigma(i)}$ for each $1 \leq i \leq m$.

Furthermore, a set $S \subset\{1,2, \ldots, 2 n\}$ is called minimal strongly array admissible for $2 n$, if $S$ is strongly array admissible for $2 n$, and for each $S^{\prime} \subset S$ with $S^{\prime} \neq S$ we have that $\operatorname{lcm}\left(S^{\prime}\right)<\operatorname{lcm}(S)$. Let $(L,<)$ be a finite totally ordered set and $L^{\prime}$ be a subset of $L$ with the ordering inherited from $L$. Now observe that if $\vartheta=\left(\vartheta_{i}: \mathbb{Z} \rightarrow \Sigma \mid i \in L\right)$ is a strongly admissible array on $2 n$ symbols, then $\vartheta^{\prime}=\left(\vartheta_{i}: \mathbb{Z} \rightarrow \Sigma \mid i \in L^{\prime}\right)$ is also a strongly admissible array on $2 n$ symbols. The array $\vartheta^{\prime}$ is called a subarray of $\vartheta$. By using this observation it is not hard to verify that

$$
\begin{aligned}
T(n) & =\{\operatorname{lcm}(S): S \text { is strongly array admissible for } 2 n\} \\
& =\{\operatorname{lcm}(S): S \text { is minimal strongly array admissible for } 2 n\} .
\end{aligned}
$$

Let us now prove the first property.

Lemma 5.1 Suppose that $\left(\vartheta_{i}: \mathbb{Z} \rightarrow \Sigma \mid i \in L\right)$ is a strongly admissible array on $2 n$ symbols, and let $p_{i}$ denote the period of $\vartheta_{i}$ for each $i \in L$. If there exist distinct $i, j \in L$ such that $\operatorname{gcd}\left(\left\{p_{i}, p_{j}\right\}\right)=1$, then

$$
\overline{R\left(\vartheta_{i}\right)} \cap \overline{R\left(\vartheta_{j}\right)}=\emptyset \text {. }
$$

Proof. Let $\vartheta=\left(\vartheta_{i}: \mathbb{Z} \rightarrow\{1, \ldots, 2 n\} \mid i \in L\right)$ be a strongly admissible array on $2 n$ symbols and let $p_{i}$ denote the period of $\vartheta_{i}$ for each $i \in L$. Suppose there exist distinct $i, j \in L$ such that $\operatorname{gcd}\left(\left\{p_{i}, p_{j}\right\}\right)=1$, and assume by way of contradiction that

$$
\overline{R\left(\vartheta_{i}\right)} \cap \overline{R\left(\vartheta_{j}\right)} \neq \emptyset .
$$

Then there exist $s, t \in \mathbb{Z}$ such that $\vartheta_{i}(s)=\vartheta_{j}(t)$ or $\vartheta_{i}(s)=\vartheta_{j}(t)^{+}$. Put $\rho=\operatorname{gcd}\left(\left\{p_{i}, p_{j}\right\}\right)$. As $\rho=1$ we have that $t-s \equiv 0 \bmod \rho$. However, if $\vartheta_{i}(s)=\vartheta_{j}(t)$ this contradicts property (ii) in Definition 1.1. On the other hand if $\vartheta_{i}(s)=\vartheta_{j}(t)^{+}$, then it contradicts property (i) in Definition 2.2. Therefore the intersection in (22) is empty.

This lemma has the following consequence. 
Corollary 5.1 If $S \subset\{1,2, \ldots, 2 n\}$ and there exist distinct $p_{1}, \ldots, p_{k} \in S$ such that $\operatorname{gcd}\left(\left\{p_{i}, p_{j}\right\}\right)=1$ for $1 \leq i<j \leq k$ and

$$
\sum_{i=1}^{k} 2\left\lceil\frac{p_{i}}{2}\right\rceil>2 n
$$

then $S$ is not strongly array admissible for $2 n$. Here $\lceil a\rceil$ denotes the smallest integer $k$ with $a \leq k$.

Proof. Suppose to the contrary that $S \subset\{1,2, \ldots, 2 n\}$ is strongly array admissible for $2 n$ and let $p_{1}, \ldots, p_{k} \in S$ be such that $\operatorname{gcd}\left(\left\{p_{i}, p_{j}\right\}\right)=1$ for $1 \leq i<j \leq k$ and

$$
\sum_{i=1}^{k} 2\left\lceil\frac{p_{i}}{2}\right\rceil>2 n
$$

Hence there exists a strongly admissible array $\left(\vartheta_{i}: \mathbb{Z} \rightarrow\{1, \ldots, 2 n\} \mid i \in L\right)$ on $2 n$ symbols such that after a possible renaming of the elements of $L$ the map $\vartheta_{i}$ has period $p_{i}$ for $1 \leq i \leq k$. From Lemma 5.1 it follows that

$$
2 n<\sum_{i=1}^{k} 2\left\lceil\frac{p_{i}}{2}\right\rceil \leq \sum_{i=1}^{k}\left|\overline{R\left(\vartheta_{i}\right)}\right|=\left|\bigcup_{i=1}^{k} \overline{R\left(\vartheta_{i}\right)}\right| \leq 2 n,
$$

which is clearly a contradiction.

By using this corollary one can show that $T(n)$ is strictly smaller that $Q(2 n)$ for each $n \geq 4$. Indeed, for each $n \geq 4$ there exist integers $n_{1}, n_{2} \in \mathbb{N}$, with $n_{1}>1$ and $n_{2}>1$, such that $n_{1}+n_{2}=2 n$ and $\operatorname{gcd}\left(\left\{n_{1}, n_{2}\right\}\right)=1$. If $n$ is even one can take $n_{1}=n-1$ and $n_{2}=n+1$, and if $n$ is odd one can take $n_{1}=n-2$ and $n_{2}=n+2$. It is easy to see that $\operatorname{lcm}\left(\left\{n_{1}, n_{2}\right\}\right) \in Q(2 n)$, but as $n_{1}$ and $n_{2}$ are both odd it follows from Corollary 5.1 that $\operatorname{lcm}\left(\left\{n_{1}, n_{2}\right\}\right)$ is not in $T(n)$.

Another property is the following.

Lemma 5.2 If $S \subset\{1,2, \ldots, 2 n\}$ and there exist distinct $p_{1}, p_{2} \in S$ with $p_{1}+p_{2}>2 n$ and $\operatorname{gcd}\left(\left\{p_{1}, p_{2}\right\}\right)=2$, then $S$ is not strongly array admissible for $2 n$.

Proof. Suppose to the contrary that $S \subset\{1,2, \ldots, 2 n\}$ is strongly array admissible for $2 n$, and assume that $p_{1}, p_{2} \in S$ distinct are such that $\operatorname{gcd}\left(\left\{p_{1}, p_{2}\right\}\right)=2$ and $p_{1}+p_{2}>2 n$. Let $L=\{1,2\}$ be equipped with the 
usual ordering. Then there exists a strongly admissible array $\left(\vartheta_{i} \mid i \in L\right)$ on $2 n$ symbols such that $\vartheta_{1}$ and $\vartheta_{2}$ have period $p_{1}$ and $p_{2}$ respectively.

For each $i \in L$ and $k \in\{1, \ldots, n\}$ let $R_{k}\left(\vartheta_{i}\right)=\left\{m \in R\left(\vartheta_{i}\right): m=\right.$ $k$ or $\left.m=k^{+}\right\}$. As

$$
\sum_{k=1}^{n}\left|R_{k}\left(\vartheta_{1}\right)\right|+\left|R_{k}\left(\vartheta_{2}\right)\right|=\left|R\left(\vartheta_{1}\right)\right|+\left|R\left(\vartheta_{2}\right)\right|=p_{1}+p_{2}>2 n,
$$

there exists $k \in\{1, \ldots, n\}$ such that $\left|R_{k}\left(\vartheta_{1}\right)\right|+\left|R_{k}\left(\vartheta_{1}\right)\right| \geq 3$. Therefore one can find $k^{\prime} \in\{1,2, \ldots, 2 n\}$ and $s, t, u \in \mathbb{Z}$ such that

$$
k^{\prime}=\vartheta_{1}(s)=\vartheta_{1}(t)^{+}=\vartheta_{2}(u) \text { or } k^{\prime}=\vartheta_{2}(s)=\vartheta_{2}(t)^{+}=\vartheta_{1}(u) .
$$

Suppose that we are in the first case. Since $\operatorname{gcd}\left(\left\{p_{1}, p_{2}\right\}\right)=2$ property (i) in Definition 2.2 yields that $u-t \not \equiv 0 \bmod 2$. Likewise property (ii) in Definition 1.1 yields that $u-s \not \equiv 0 \bmod 2$. Hence $u-t \equiv u-s \equiv 1 \bmod 2$, which contradicts property (ii) in Definition 2.2. Analogously one can derive a contradiction in the second case. Thus, we conclude that $S$ is not strongly array admissible for $2 n$.

A similar result is the following.

Lemma 5.3 If $S \subset\{1,2, \ldots, 2 n\}$ and there exist distinct $p_{1}, p_{2} \in S$ with $p_{1}+p_{2}>3 n$ and $\operatorname{gcd}\left(\left\{p_{1}, p_{2}\right\}\right)=3$, then $S$ is not strongly array admissible for $2 n$.

Proof. Suppose to the contrary that $S \subset\{1,2, \ldots, 2 n\}$ is strongly array admissible for $2 n$, and assume that there are distinct $p_{1}, p_{2} \in S$ such that $\operatorname{gcd}\left(\left\{p_{1}, p_{2}\right\}\right)=3$ and $p_{1}+p_{2}>3 n$. Let $L=\{1,2\}$ be equipped with the usual ordering. Then there exists a strongly admissible array $\left(\vartheta_{i} \mid i \in L\right)$ on $2 n$ symbols such that $\vartheta_{1}$ and $\vartheta_{2}$ have period $p_{1}$ and $p_{2}$ respectively.

For each $i \in L$ and $k \in\{1, \ldots, n\}$ let $R_{k}\left(\vartheta_{i}\right)=\left\{m \in R\left(\vartheta_{i}\right): m=\right.$ $k$ or $\left.m=k^{+}\right\}$. As

$$
\sum_{k=1}^{n}\left|R_{k}\left(\vartheta_{1}\right)\right|+\left|R_{k}\left(\vartheta_{2}\right)\right|=\left|R\left(\vartheta_{1}\right)\right|+\left|R\left(\vartheta_{2}\right)\right|=p_{1}+p_{2}>3 n,
$$

there exists $k \in\{1, \ldots, n\}$ such that $\left|R_{k}\left(\vartheta_{1}\right)\right|+\left|R_{k}\left(\vartheta_{2}\right)\right|=4$. This implies that there exist $s, t, v, w \in \mathbb{Z}$ such that $\vartheta_{1}(s)=\vartheta_{1}(t)^{+}=\vartheta_{2}(v)=\vartheta_{2}(w)^{+}$.

It follows from Property (ii) in Definition 1.1 that

$$
v-s \not \equiv 0 \bmod 3 \text { and } w-t \not \equiv 0 \bmod 3 .
$$


Similarly, property (i) in Definition 2.2 yields that

$$
w-s \not \equiv 0 \bmod 3 \text { and } v-t \not \equiv 0 \bmod 3 .
$$

Moreover property (ii) in Definition 2.2 implies: $v-s \not \equiv w-s$ mod 3 and $w-t \not \equiv w-s \bmod 3$, so that (23) and (24) yield $v-s \equiv w-t \bmod 3$.

Now there are two cases, namely $v-s \equiv 1 \bmod 3$ and $v-s \equiv 2 \bmod 3$. In the first case we have that $v-t \equiv(v-s)-(w-s)+(w-t) \equiv 1-$ $2+1 \equiv 0 \bmod 3$, which contradicts $(24)$. Likewise, $v-s \equiv 2 \bmod 3$ implies $v-t \equiv(v-s)-(w-s)+(w-t) \equiv 2-1+2 \equiv 0 \bmod 3$, which again contradicts (24). Thus $S$ is not strongly array admissible for $2 n$.

More properties of this type can be found in [3, Section 3.5] by the author. Let us now use the results from this section to make several remarks concerning the optimality of the upper estimate $T(n)$ for $R(n)$.

\section{Remarks concerning the optimality of the esti- mate}

A detailed analysis of how well $T(n)$ approximates $R(n)$ for $1 \leq n \leq 10$ is given by the author in [3, Section 3.6]. In this section we will shortly discuss the results from that work. It is not hard to see that $R(1)=T(1)=$ $Q(2)=\{1,2\}$ and that $R(2)=T(2)=Q(4)=\{1,2,3,4\}$ (cf. [3, Section 3.6]). However, if the dimension is 3 some more work is needed.

Theorem 6.1 $R(3)=T(3)=\{1,2,3,4,5,6\}$.

Proof. It is shown in [3, Section 3.5] that $\{1,2,3,4,5,6\} \subset R(3)$. Furthermore one can find in [13] that $Q(6)=\{1,2,3,4,5,6,12\}$. As $R(3) \subset T(3) \subset$ $Q(6)$ it suffices to show that 12 is not in $T(3)$.

Remark that there are only two candidate minimal strongly array admissible sets for 6 that will give period 12 , namely $S_{1}=\{3,4\}$ and $S_{2}=\{4,6\}$. The set $S_{1}$ is not strongly array admissible for 6 by Corollary 5.1. Lemma 5.2 yields that $S_{2}$ is not strongly array admissible for 6 . Hence $12 \notin T(3)$, so that $R(3)=T(3)=\{1,2,3,4,5,6\}$. $n=4$.

This proves the conjecture in [12, page 171]. Let us now discuss the case

Theorem $6.2 R(4)=T(4)=\{1,2,3,4,5,6,7,8,10,12\}$. 
Proof. In [13] we find $Q(8)=\{1,2,3,4,5,6,7,8,10,12,15,24\}$. Furthermore it is shown in $[3$, Section 3.5] that $\{1,2,3,4,5,6,7,8,10,12\} \subset R(4)$. Since $R(4) \subset T(4) \subset Q(8)$ it is sufficient to show that 15 and 24 are both not in $T(4)$.

To obtain period 15 there is precisely one candidate minimal strongly array admissible set for 8 , namely $S=\{3,5\}$. It follows from Corollary 5.1 however, that $S$ is not strongly array admissible for 8 .

Now consider period 24 . In this case there are two candidate sets, namely $S_{1}=\{3,8\}$ and $S_{2}=\{6,8\}$. The set $S_{1}$ is not strongly array admissible for 8 by Corollary 5.1. It follows from Lemma 5.2 that $S_{2}$ is not strongly array admissible for 8 . Therefore we conclude that $R(4)=T(4)=\{1,2,3,4,5,6,7,8,10,12\}$.

A similar analysis can be used for the dimensions $5 \leq n \leq 10$. The results are summarized in the following theorem. For a proof we refer the reader to [3, Section 3.6].

Theorem 6.3 The following assertions are true:

(1) $R(n)=T(n)$ for $n=1,2,3,4,6,7,10$.

(2) $T(5) \backslash\{18\} \subset R(5) \subset T(5)$.

(3) $T(8) \backslash\{90\} \subset R(8) \subset T(8)$.

(4) $T(9) \backslash\{126\} \subset R(9) \subset T(9)$.

For $1 \leq n \leq 10$ a complete list of the elements of the set $T(n)$ is given at the end of the paper in Table 1. From Theorem 6.3 it follows that up to $n=10$ the set $R(n)$ is completely determined with the exception of three integers: 18,90 , and 126. Let us take a closer look at these integers.

To generate period 18 in $T(5)$ there are two candidate minimal strongly array admissible sets, namely $S_{1}=\{2,9\}$ and $S_{2}=\{6,9\}$. By using Corollary 5.1 we see that $S_{2}$ is actually the only possible set. An example of a strongly admissible array on 10 symbols $\left(\vartheta_{i}: \mathbb{Z} \rightarrow\{1, \ldots, 10\} \mid i=1,2\right)$, where $\vartheta_{1}$ and $\vartheta_{2}$ have period 9 and 6 respectively, can be given as follows. Let $L=\{1,2\}$ be equipped with the usual ordering and let $\vartheta_{1}: \mathbb{Z} \rightarrow\{1, \ldots, 10\}$ be defined by

\begin{tabular}{rlllllllll}
$s:$ & 0 & 1 & 2 & 3 & 4 & 5 & 6 & 7 & 8 \\
\hline$\vartheta_{1}(s):$ & 10 & 1 & 2 & 3 & 4 & 5 & 7 & 8 & 9
\end{tabular}


and for general $s \in \mathbb{Z}$ let $\vartheta_{1}(s)=\vartheta_{1}\left(s^{*}\right)$, where $0 \leq s^{*}<9$ and $s \equiv s^{*} \bmod 9$. The map $\vartheta_{2}: \mathbb{Z} \rightarrow\{1, \ldots, 10\}$ is defined as follows:

$$
\begin{array}{rllllll}
s: & 0 & 1 & 2 & 3 & 4 & 5 \\
\hline \vartheta_{2}(s): & 1 & 2 & 3 & 4 & 5 & 6
\end{array}
$$

and for general $s \in \mathbb{Z}$ we let $\vartheta_{2}(s)=\vartheta_{2}\left(s^{*}\right)$, where $0 \leq s^{*}<6$ and $s \equiv$ $s^{*} \bmod 6$.

These observations have the following consequence. If there exists a lower semilattice homomorphism $f: V \rightarrow V$, with $V \subset \mathbb{E}^{10}$, which has a periodic point $\xi$ of minimal period 18 , then every complete sequence for $\xi$ contains two points that have minimal period 6 and 9 under $f$. For the periods 90 and 126 one can also prove that they both have a unique minimal strongly array admissible set namely: $\{5,6,9\}$ and $\{6,7,9\}$, respectively.

\section{Concluding remarks}

In this paper we have introduced a set $T(n)$, for $n \in \mathbb{N}$, that is determined by arithmetical and combinatorial constraints. As a main result we proved in Theorem 2.1 that $R(n) \subset T(n)$ for all $n \in \mathbb{N}$. After a further analysis of the set $T(n)$ we were able to show that $R(n)=T(n)$ for $n=1,2,3,4,6,7$, and 10 (see Theorem 6.3). For all other $n \in \mathbb{N}$ however, it remains unknown if the sets $R(n)$ and $T(n)$ are equal.

problem 7.1 Decide whether $R(n)=T(n)$ for all $n \in \mathbb{N}$.

To obtain the set $R(n)$ up to $n=10$ it suffices to know if $18 \in R(5)$, $90 \in R(8)$, and $126 \in R(9)$ (see Theorem 6.3). As $5 \in R(3)$ and $7 \in R(4)$ we see that $18 \in R(5)$ implies $90 \in R(8)$ and $126 \in R(9)$. This gives some extra motivation to decide whether or not 18 is in $R(5)$.

A combination of Corollary 3.1 and Theorem 3.2 yields that for each $p \in R(n)$ there exist a lower semilattice $V \subset \mathbb{E}^{2 n}$ and a lower semilattice homomorphism $g: V \rightarrow V$, which has a periodic point of $\operatorname{minimal}$ period $p$. Therefore, if $Q^{*}\left(\mathbb{E}^{2 n}\right)$ denotes the set of possible minimal periods of periodic points of lower semilattice homomorphisms $g: V \rightarrow V$ with $V \subset \mathbb{E}^{2 n}$, then $R(n) \subset Q^{*}\left(\mathbb{E}^{2 n}\right)$. From Proposition 4.1 it follows that

$$
R(n) \subset Q^{*}\left(\mathbb{E}^{2 n}\right) \subset T(n) \text { for all } n \in \mathbb{N} .
$$

A more philosophical problem would be to find out if one of the inclusions in $(25)$ can be replaced by an equality. In particular, it would be interesting to know if $18 \in Q^{*}\left(\mathbb{E}^{10}\right)$. 
Table 1: The elements of $T(n)$ for $1 \leq n \leq 10$.

\begin{tabular}{ll}
\hline$n$ & Elements of $T(n)$ \\
\hline 1 & 1,2 \\
2 & $1,2,3,4$ \\
3 & $1,2,3,4,5,6$ \\
4 & $1,2,3,4,5,6,7,8,10,12$ \\
5 & $1,2,3,4,5,6,7,8,9,10,12,14,15,18,20$ \\
6 & $1,2,3,4,5,6,7,8,9,10,11,12,14,15,18,20,21,24,28,30$ \\
7 & $1,2,3,4,5,6,7,8,9,10,11,12,13,14,15,18,20,21,22,24$, \\
& $28,30,35,36,40,42,60$ \\
8 & $1,2,3,4,5,6,7,8,9,10,11,12,13,14,15,16,18,20,21,22$, \\
& $24,26,28,30,33,35,36,40,42,44,45,48,56,60,70,84,90$ \\
9 & $1,2,3,4,5,6,7,8,9,10,11,12,13,14,15,16,17,18,20,21$, \\
& $22,24,26,28,30,33,35,36,39,40,42,44,45,48,52,55,56$, \\
& $60,63,66,70,72,84,90,105,120,126,140$ \\
10 & $1,2,3,4,5,6,7,8,9,10,11,12,13,14,15,16,17,18,19,20$, \\
& $21,22,24,26,28,30,33,34,35,36,39,40,42,44,45,48,52$, \\
& $55,56,60,63,65,66,70,72,77,78,80,84,88,90,105,110$, \\
& $120,126,132,140,168,180,210$
\end{tabular}

\section{References}

[1] M. A. Akcoglu and U. Krengel. Nonlinear models of diffusion on a finite space. Probab. Theory Related Fields 76 (1987), 411-420.

[2] A. Blokhuis and H.A. Wilbrink. Alternative proof of Sine's theorem on the size of a regular polygon in $\mathbb{R}^{k}$ with the $\ell_{\infty}$ metric. Discrete Comput. Geom. 7 (1992), 433-434.

[3] B. Lemmens. Iteration of nonexpansive maps under the 1-norm. PhD. thesis. (Vrije Universiteit Amsterdam, Amsterdam, The Netherlands, June 2001).

[4] B. Lemmens, R.D. Nussbaum, and S.M. Verduyn Lunel. Lower and upper bounds for $\omega$-limit sets of nonexpansive maps. Indag. Mathem. (N.S.). To appear.

[5] R. Lyons and R.D. Nussbaum. On transitive and commutative finite groups of isometries. In Fixed Point Theory and Applications, K.-K. Tan, Ed., (World Scientific, Singapore, 1992), 189-228. 
[6] P. Martus. Asymptotic properties of nonstationary operator sequences in the nonlinear case. PhD. thesis. (Friedrich-Alexander Univ., Erlangen-Nürnberg, Germany, November 1989). In German.

[7] M. Misiurewicz. Rigid sets in finite dimensional $l_{1}$-spaces. Tech. Rep. 45, Mathematica Göttingensis Schriftenreihe des Sonderforschungsbereichs Geometrie und Analysis (1987).

[8] R. D. Nussbaum. Omega limit sets of nonexpansive maps: finiteness and cardinality estimates. Differential Integral Equations 3 (1990), 523540 .

[9] R. D. Nussbaum. Estimates of the periods of periodic points of nonexpansive operators. Israel J. Math. 76 (1991), 345-380.

[10] R. D. Nussbaum. A nonlinear generalization of Perron-Frobenius theory and periodic points of nonexpansive maps. In Recent Developments in Optimization and Nonlinear Analysis Contemporary Mathematics vol. 204, Y. Censor and S. Reich, Eds., (American Mathematical Society, Providence, R.I., 1997), 183-198.

[11] R. D. Nussbaum and M. Scheutzow. Admissible arrays and a generalization of Perron-Frobenius theory. J. London Math. Soc. (2) 58 (1998), 526-544.

[12] R.D. Nussbaum, M. Scheutzow, and S.M. Verduyn Lunel. Periodic points of nonexpansive maps and nonlinear generalizations of the Perron-Frobenius theory. Selecta Math. (N.S.) 4 (1998) 1-41.

[13] R. D. Nussbaum and S.M. Verduyn Lunel. Generalizations of the Perron-Frobenius theorem for nonlinear maps. Mem. Amer. Math. Soc. (138) 659 (1999), 1-98.

[14] M. Scheutzow. Periods of nonexpansive operators on finite $l_{1}$-spaces. European J. Combin. 9 (1988), 73-78.

[15] M. Scheutzow. Corrections to periods of nonexpansive operators on finite $l_{1}$-spaces. European J. Combin. 12 (1991), 183.

[16] R. Sine. A nonlinear Perron-Frobenius theorem. Proc. Amer. Math. Soc. 109 (1990), 331-336.

[17] D. Weller. Hilbert's metric, part metric and self mappings of a cone. PhD. thesis. (Universität Bremen, Germany, December 1987). 